\title{
PERFIL EPIDEMIOLÓGICO DO HIV EM UM PEQUENO MUNICÍPIO POTIGUAR / BRASIL, ENTRE 2015 E 2018
}

\section{EPIDEMIOLOGICAL PROFILE OF HIV IN A SMALL MUNIPALITY POTIGUAR / BRAZIL, BETWEEN 2015 AND 2018}

\author{
Gislanne Stéphanne Estevam da Silva1, Franklin Learcton Bezerra de Oliveira², Dany Geraldo Kramer ${ }^{3}$
}

\begin{abstract}
RESUMO
Introdução: O acompanhamento da incidência de HIV na população se faz importante para poder se prever as taxas futuras de novas infecções pelo HIV em uma comunidade em particular. Desta forma ações e programas de prevenção e controle podem ser realizadas ou planejadas. Objetivo: Nesta conjuntura, o presente estudo objetivou caracterizar o perfil epidemiológico de pessoas diagnosticadas com HIV, entre os anos de 2015 e 2018, em um pequeno município do Rio Grande do Norte, Brasil. Método: Trata-se de uma pesquisa descritiva e exploratória desenvolvida a partir de informações coletadas sobre os casos notificados e confirmados de HIVIAIDS, através do acesso aos dados do Sistema de Informações de Agravos e Notificações (SINAN) entre os anos de 2015 a 2018. Resultados: Através do presente estudo observou que maioria dos infectados foram do masculino. Além disso, informações relevantes, como escolaridade não foram notificadas. Conclusões: Desta forma, se faz necessário que sejam criadas estratégias por meio do serviço público que atinjam essa população no quesito prevenção e orientação aos profissionais sobre a importância dos registros adequados.
\end{abstract}

PALAVRAS-CHAVES: HIV; Perfil epidemiológico; Pequeno Município; Brasil.

\begin{abstract}
Monitoring the incidence of HIV in the population is important in order to predict future rates of new HIV infections in a particular community. In this way prevention and control actions and programs can be carried out or planned. At this juncture, the present study aimed to characterize the epidemiological profile of people diagnosed with HIV between 2015 and 2018 in a small municipality of Rio Grande do Norte, Brazil. This is a descriptive and exploratory research based on information collected on the reported and confirmed cases of HIV / AIDS, through access to data from the Aging and Notification Information System (SINAN) between the years 2015 to 2018. Through the present study it was observed that the majority of the infected were male. In addition relevant information such as schooling was not reported. Thus, it is necessary to create strategies through the public service that reach this population in the prevention and guidance to professionals on the importance of adequate records.
\end{abstract}

KEYWORDS: HIV; Epidemiological profile; Small Town; Brazil.

Discente - Faculdade de Ciências da Saúde do Traíri - UFRN.

2 Enfermeiro; Mestre em Enfermagem pela UFRN; Docente da Escola Técnica do SUS; Enfermeiro da Estratégia de Saúde da Família de Equador/RN.

3 Prof. Dr. Faculdade, de Ciências da Saúde do Traíri - UFRN. Mestrado Em Saúde da Família - UFRN. Av. Rio Branco, S/N, Centro, Santa Cruz / RN. FACISA. 59200-000. dgkcs@yahoo.com.br. 8432912411. 


\section{INTRODUÇÃO}

A Síndrome da Imunodeficiência Adquirida (AIDS) tem sido denominada como a mais recente pandemia da humanidade, constitui-se atualmente um dos mais sérios problemas mundiais de saúde pública. É uma doença crônica causada pelo Vírus da Imunodeficiência Humana (HIV), ocasionando perda progressiva da imunidade celular e, consequentemente, o aparecimento de infecções oportunistas. Existem várias vias de transmissão (ocupacional, vertical, em usuários de drogas injetáveis), mas a via sexual é considerada a mais comum ${ }^{1,2}$.

No Brasil, os primeiros casos de HIVIAIDS surgiram na década de 1980, em que houve implantação de políticas públicas na prevenção dessa infecção e de retrovirais que ocasionaram mudanças no perfil epidemiológico. Desde dessa época, vários estudos estão sendo realizados por profissionais da saúde para aperfeiçoamento na prevenção, diagnóstico, tratamento, adoecimento e morte por pessoas infectadas pelo vírus ${ }^{3-5}$.

Em anos recentes, foi implementado na atenção primária à saúde, a partir da estratégia de saúde da família, os testes rápidos para prevenção das infecções sexualmente transmissíveis, dentre elas o HIV, que faz parte da meta global em resposta à epidemia do HIV5-7.

No mundo, em 2017, aproximadamente 1 (um) milhão e 800 (oitocentas) mil pessoas foram infectadas pelo HIV, 36.900 .000 (trinta e seis milhões e 900 mil) pessoas vivem com o vírus, sendo que 21.700.000 (vinte e um milhões e setecentos mil) vivendo em tratamento com antirretrovirais. Enquanto isso, em 2017, aproximadamente 866 mil pessoas viviam com HIV no Brasil, das quais 731 mil estavam diagnosticadas, sendo 509 mil homens e 307 mil mulheres ${ }^{7-9}$.

Segundo dados do Ministério da Saúde, no Rio Grande do Norte 6.420 pessoas que vivem com HIV (PVHIV) fazem tratamento com antirretrovirais, sendo o estado que se destaca seguido da Bahia. No que se refere a supressão, das PVHIV vinculadas, as maiores proporções foram observadas na BA $(72 \%)$, no RN (71\%) e no Ceara (CE), com $70 \%{ }^{7}$.

Em cidades menores, do interior brasileiro, como a região do Traíri - Rio Grande do Norte, o fenômeno de interiorização do HIV/aids, envolvem municípios com menor renda per capita, com pessoas de baixa renda, habitações precárias, dificuldades no acesso a serviço público de saúde e transportes o que pode comprometer, às consultas de acompanhamento, realização dos exames específicos ou programas de educação em saúde ${ }^{10-12}$.
Nesse sentido, apresentar dados regionais, principalmente de cidades menores, permite aos profissionais de saúde repensarem sobre a sua prática sobre as pessoas que vivem com HIV, pois existem grandes diferenças sexuais, econômicas, sociais e culturais no país. Este trabalho terá como objetivo caracterizar o perfil epidemiológico de pessoas diagnosticadas com HIV, entre os anos de 2015 e 2018, no município de Santa Cruz, Rio Grande do Norte, Brasil.

\section{METODOLOGIA LOCAL E CARACTERIZAÇÃO DO ESTUDO}

O estudo foi realizado no município de Santa Cruz, localizado na microrregião da Borborema Potiguar, Rio Grande do Norte, que possui uma população de aproximadamente 39 mil habitantes ${ }^{13}$. Nesta cidade há um Serviço de Atendimento Especializado (SAE) em HIV/ AIDS em um Hospital Regional, contando profissionais de enfermagem, psicologia e assistente social, onde há realização de testes rápidos e sorologias, vinculados ao Sistema Único de Saúde (SUS), sendo uma referência Regional de atendimento a este agravo.

A presente pesquisa é caracterizada como descritiva e exploratória desenvolvida a partir de informações coletadas sobre os casos notificados e confirmados de HIVIAIDS no município de Santa Cruz entre os anos de 2015 a 2018. A pesquisa exploratória se dá em fenômeno pouco ou nenhuma vez estudado, tornando essencial para elaboração de hipóteses ${ }^{14}$.

\section{COLETA DE DADOS}

A coleta de dados ocorreu através de acesso aos dados do Sistema de Informações de Agravos e Notificações (SINAN), junto ao setor de epidemiologia da Secretaria Municipal de Saúde de Santa Cruz-RN, e consentimento deste setor. Tal setor é responsável por alimentar o SINAN, dentre eles o Programa DST/AIDS, responsável pelo registro de casos de HIVIAIDS no município. Foram analisados os registros relativos ao período de janeiro de 2015 a dezembro de 2018. A pesquisa levou em consideração os registros de HIV quanto os casos notificados de AIDS e aspectos sócio-demográficos do paciente.

\section{RESULTADOS E DISCUSSÃO}

A infecção pelo HIV e a AIDS, independente da faixa etária, são de notificação compulsória e podem ser 
notificados por qualquer profissional de saúde ou responsáveis pelos estabelecimentos de saúde públicos ou privados $^{7,15,16}$.

A pesquisa resultou entre os anos de 2015 a 2018 , em 22 casos da Síndrome da Imunodeficiência Adquirida no município, sendo o ano de 2015 com maior número de casos, com 11 ao total. Além desses achados, observou-se que dos 22 casos, 15 residiam no município e os demais eram residentes de outras localidades; sendo que 17 encontravam-se na zona urbana e 5 na zona rural.

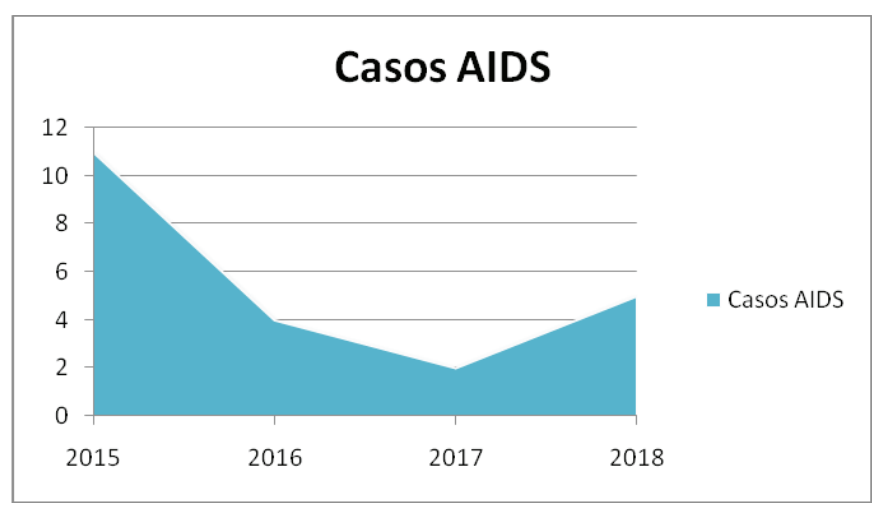

Figura 1 - Casos AIDS no município de Santa Cruz entre 2015 a 2018.

Esses casos tornam-se importantes na medida de aumentar as ações de vigilância, diagnóstico, prevenção e tratamento de pessoas que convivem com o HIV no município, além de promover o hábito manterem relações sexuais seguras e procurar os serviços de saúde para realização do teste rápido ou o Serviço de Atendimento Especializado para o tratamento. A ausência de registro de notificação provoca comprometimento na execução do sistema de vigilância, devido o comprometimento da programação orçamentária ${ }^{7,17}$.

Em relação as escolaridades, 19 fichas analisadas tiveram a escolaridade ignorada, enquanto duas pessoas eram analfabetas e um tinha ensino médio (Figura 02).

\section{Escolaridade}

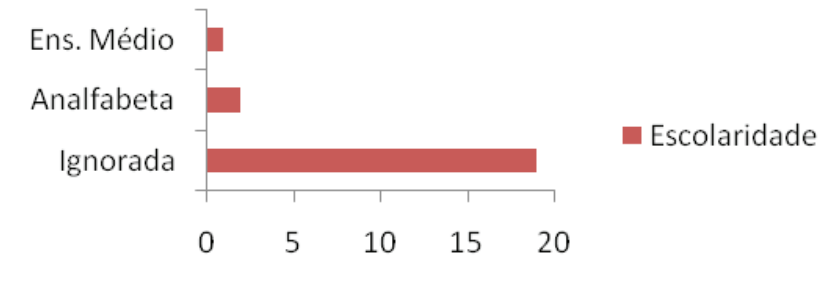

Figura 02: Nível de escolaridade dos pacientes infectados.
Ter o registro da escolaridade preenchida favorece identificar se o público que está sendo infectado tinha ou não conhecimento sobre a doença e suas consequências. Além disso, outro achado com uma quantidade significativa sem registro foi à raça. Os dados registrados pelos profissionais devem ser todos preenchidos para que, em algum momento, possam fazer uma caracterização e aplicar ações de intervenção que condizem com sua realidade ${ }^{2,18,19}$.

Nenhuma ação isolada irá reduzir os números de infecção pelo HIV, pois existem diferentes fatores de risco, exposição e transmissão, na mais diversidade cultural, econômica, social e política do território brasileiro. Dessa forma, a prevenção combinada, aquela que une intervenções biomédicas, estruturais e comportamentais, devem ser executadas de acordo com a realidade do indivíduo, respeitando suas necessidades especiais e as variadas formas de transmissão do vírus ${ }^{20-22}$

O sexo predominante na infecção pelo HIV é o masculino com 13 casos contra o feminino com 9 . Esses dados estão em consonância com achados nacionais de 2017, em que aproximadamente 559 mil homens estavam infectados pelo HIV, já as mulheres eram $307 \mathrm{mil}^{4,7}$.

Com relação à infecção pelo HIV em gestantes, no município foram notificados 19 casos, dos quais 17 não residiam no município, tendo 17 registros com escolaridade ignorada e duas com ensino fundamental incompleto. No mesmo caminho, sobre a raça, 16 não tinham registros; já com relação à faixa etária, esta variou de 30 a 40 anos de idade. O Ministério da Saúde orienta os profissionais para que na primeira consulta seja realizado os testes rápidos para HIV, Sífilis e Hepatites B e C, para identificação e intervenção com objetivo de evitar a transmissão dessas doenças da mãe para o feto ${ }^{7,23}$.

Os dados pesquisados mostraram que 15 crianças estavam expostas ao vírus do HIV, das quais todas residiam na zona urbana do município, sendo 10 crianças da raça parda e 5 da raça branca. Diante desses casos, é primordial que os profissionais de saúde orientem a mãe portadora do HIV sobre a necessidade de apoio, segurança e tratamento do vírus, para que saiba lidar com a doença e reduzir os riscos de transmissão vertical ao seu filho. A equipe de saúde é responsável pelos cuidados desempenhados pelos familiares à criança, promovendo hábitos que conscientize e compreenda toda a dinâmica da infecção pelo HIV ${ }^{15,24,25}$. 
A identificação precoce de crianças expostas ao HIV ou infectada verticalmente é de suma importância para indicar o início do tratamento com antirretrovirais e ações profiláticas para infecções oportunistas, além de outras manifestações clínicas ${ }^{7,26,27}$.

Portanto, acompanhamentos epidemiológicos observacionais deste tipo fornecem dados valiosos para o desenho e a conduta dos ensaios de prevenção, planejamento e prestação de serviços. Dessa forma que, a incidência possa ser reduzida ao longo dos próximos períodos, acarretando em benefícios para a saúde pública, comunidades científicas e locais, que são enormes.

\section{CONCLUSÕES}

Através do presente estudo observou que maioria dos infectados foram do masculino. Além disso informações relevantes, como escolaridade não foram notificadas. Dessa forma, se faz necessário que sejam criadas estratégias por meio do serviço público que atinjam essa população no quesito prevenção e orientação aos profissionais sobre a importância dos registros adequados.

Esses dados têm importantes implicações para a saúde pública para o desenvolvimento de futuras intervenções de prevenção do HIV principalmente entre os jovens. 


\section{REFERÊNCIAS}

1. Ghys PD, Williams BG, Over M, Hallett TB, Godfrey-Faussett P. Epidemiological metrics and benchmarks for a transition in the HIV epidemic. PLoS Med. [internet]. 2018 [acesso em 2019 out 31];15(10): e1002678. Disponible en: https://doi. org/10.1371/journal.pmed.1002678

2. Whiteside A, Vinnitchok A, Dlamini T, Mabuza K. Mixed results: the protective role of schooling in the HIV epidemic in Swaziland. Afr $\mathrm{J}$ AIDS Res. 2017 Dec; 16(4):305-313. doi: 10.2989/16085906.2017.1362016.

3. Barros SG, Vieira-da-Silva LM. A gênese da política de luta contra a aids e o Espaço Aids no Brasil (1981-1989). Rev Saude Publica. 2016;50:43.4.

4. Saffier IP, Kawa H, Harling G. A scoping review of prevalence, incidence and risk factors for HIV infection amongst young people in Brazil. BMC Infect Dis. 2017; 17(1):675. doi: 10.1186/s12879-017-2795-9.

5. Mangal TD, Pascom ARP, Vesga JF, Meireles MV, Benzaken AS, Hallett TB. Estimating HIV incidence from surveillance data indicates a second wave of infections in Brazil. Epidemics. [internet]. 2019 [acesso em 2019 out 31];27:77-85. Disponible en: https://doi.org/10.1016/i.epidem.2019.02.002.

6. UNAIDS. 90-90-90: An ambitious treatment target to help end the AIDS epidemic. Joint United Nations Programme on HIVIAIDS (UNAIDS); 2014.

7. Brasil. Ministério da Saúde. Secretaria de Vigilância em Saúde. Departamento de DST, Aids e Hepatites Virais. Protocolo clínico e diretrizes terapêuticas para manejo da infecção pelo HIV em crianças e adolescentes; 2018. $4^{\circ}$ edição. Brasília.

8. Araújo TME, Dias SRS, Sousa KAA, Silva AAS. Vulnerabilidade de pessoas privadas de liberdade ao virus da imunodeficiência humana. Rev Cubana Enferm. [Internet]. 2019 [acesso em 2019 nov 8];34(4):[aprox. 0 p.]. Disponible en: http://www.revenfermeria.sld.cu/index.php/enf/article/view/1571
9. Caro-Vega Y, Belaunzarán-Zamudio PF, Crabtree-Ramírez B, Shepherd BE, Mejia F, Giganti MJ, et al. Trends in proportion of older HIV-infected people in care in Latin America and the Caribbean: a growing challenge. Epidemiology and Infection. 2018; 146(10):1308-11. doi:10.1017/S0950268818001346

10. Silva JVF, junior FJMN, Rodrigues APRA. Fatores de não adesão ao tratamento antirretroviral: desafio de saúde pública. Cadernos de Graduação: Ciências Biológicas e da Saúde. 2014; 2(1):165-75.

11. Fonseca JSA, David HMSL, Silva TFD, Ramos TCDS, Neves ACL, Miranda RB. Social networks, access and regulation of health services in a small city in the state of Rio de Janeiro, Brazil. Cien Saude Colet. 2018 Oct; 23(10):3211-3222. doi: 10.1590/1413-812320182310.15492018.

12. Miranda WA, Medeiros LB, Nascimento JÁ, Ribeiro KSQS, Nogueira JÁ, Leadebal ODCP. Modelo preditivo de retenção no cuidado especializado em HIV/aids. Cad. Saúde Pública [Internet]. 2018 [acesso em 2019 nov 8];34(10): e00209416. Disponible en: http://www.scielo.br/scielo.php?script=sci arttext\&pid=S0102-311X2018001005014\&Ing=en

13. IBGE, Instituto Brasileiro de Geografia e Estatística. Senso demográfico - Cidades: Santa Cruz; 2019.

14. Nardi PM. Doing survey research: a guide to quantitative methods; 2018. 4th Edition, 272 pages. New York.

15. Tanaka LF, Latorre MRDO, Silva AM, Konstantyner TCRO, Mendes EC, Marques HHS. Poor diet quality among Brazilian adolescents with HIVIAIDS. Jornal de Pediatria [internet]. 2015 [acesso em 2019 out 31]; 91(2):152-159. Disponível em: https://dx.doi. org/10.1016/j.jped.2014.06.007

16. Maia DAC, Zanin L, Silva ASV, Ambrosano GMB, Flório FM. Notificação de casos de HIVIAIDS em idosos no estado do Ceará: série histórica entre os anos de 2005 a 2014. Rev bras geriatr gerontol. 2018; 21(5): 542-552. 
17. Cruz ML, Cardoso CA, Darmont MQ, et al. Children and adolescents with perinatal HIV-1 infection: factors associated with adherence to treatment in the Brazilian context. Int J Environ Res Public Health. 2016; 13(6):615. doi:10.3390/ijerph13060615

18. Neve J, Fink G, Subramanian SV, Moyo S, Bor J. Length of secondary schooling and risk of HIV infection in Botswana: evidence from a natural experiment. Lancet Glob Health. 2015 Aug; 3(8):e470-e477. doi: 10.1016/S2214-109X(15)00087-X.

19. Cluver LD, Orkin M F, Yakubovic AR, Sherr L. Combination social protection for reducing HIV-risk behavior amongst adolescents in South Africa. J Acquir Immune Defic Syndr. 2016 May 1;72(1):96-104. doi: 10.1097/QAI.0000000000000938.

20. Dehne KL, Dallabetta G, Wilson D, Garnett GP, Laga M, Benomar E et al. HIV Prevention 2020: a framework for delivery and a call for action. Lancet HIV. 2016 Jul;3(7):e323-32. doi: 10.1016/S23523018(16)30035-2.

21. Brasil, Ministério da Saúde, Secretaria de Vigilância em Saúde, Departamento de Vigilância, Prevenção e Controle das Infecções Sexualmente Transmissíveis, do HIV/Aids e das Hepatites Virais. Boletim Epidemiológico Aids/DST; 2017, $3^{\circ}$ edição. Brasília.

22. Eisinger RW, Fauci AS. Ending the HIVIAIDS pandemic. Emerg Infect Dis. 2018; 24(3):413-416. doi:10.3201/eid2403.171797
23. Silva CM, Alves RS, Santos TS, Bragagnollo GR, Tavares CM, Santos AAP. Epidemiological overview of HIVIAIDS in pregnant women from a state of northeastern Brazil. Rev Bras Enferm. [internet]. 2018 [acesso em 2019 out 31];71(Suppl. 1):568-576. Disponible en: https://dx.doi.org/10.1590/0034-7167$\underline{\text { 2017-0495 }}$

24. Sherlock MSM, Cardoso MVL, Lopes MMCO, Lelis ALPA, Oliveira NR. Imunização em criança exposta ou infectada pelo hiv em um serviço de imunobiológicos especiais. Esc. Anna Nery. [internet]. 2011 [acesso em 2019 out 31];15(3):573-580. Disponible en: https://dx.doi.org/10.1590/S1414$\underline{81452011000300019}$

25. Frota ACC, Ferreira B, Harrison LH, Pereira GS, Pereira-Manfro W, Machado ES et al. Safety and immune response after two-dose meningococcal C conjugate immunization in HIV-infected children and adolescents in Rio de Janeiro, Brazil. Vaccine. 2017; 15;35(50):7042-7048. doi: 10.1016/j.vaccine.2017.10.043.

26. Bohlius J, Maxwell N, Spoerri A, Wainwright $R$, Sawry $S$, Poole $J$ et al. Incidence of AIDS-Defining and other cancers in HIV-Positive children in South Africa: Record Linkage Study. Pediatr Infect Dis J. 2016 Jun;35(6):e164-70. doi: 10.1097/ INF.0000000000001117.

27. Muenchhoff M, Healy M, Singh R, Roider J, Groll A, Kindra C. Malnutrition in HIV-Infected children is an indicator of severe disease with an impaired response to antiretroviral therapy. AIDS Res Hum Retroviruses. 2018 Jan;34(1):46-55. doi: 10.1089/ AID.2016.0261. 